\title{
Restricted Estimator in Two Seemingly Unrelated Regression Model
}

Funda Erdugan

Department of Statistics, Faculty of Arts and Sciences, Kırıkkale University, Turkey

ferdugan@gmail.com

Fikri AKDENIZ

Department of Mathematics and Computer Science

Faculty of Arts and Sciences

Çağ University, 33800 Mersin, Turkey.

fikriakdeniz@gmail.com

\begin{abstract}
This article is concerned with the estimation problem of multicollinearity in two seemingly unrelated regression (SUR) equations with linear restrictions. We propose a restricted feasible SUR estimates of the regression coefficients of this model and compare with feasible generalized least squares (FGLS) estimator and the estimator proposed by Revankar (1974) in the matrix mean square error sense. The ideas in the article are evaluated using Monte Carlo simulation.
\end{abstract}

Keywords: Restricted feasible estimator, Seemingly unrelated regressions, Two-stage estimator.

\section{Introduction}

The seemingly unrelated regression model, introduced by Zellner (1962) improves the estimation efficiency by combining several equations into a single equation. The SUR model has simulated a countless theoretical and empirical results in many fields, such as econometrics, industry, biological sciences and etc. Zellner (1963), Revankar (1974), Kariya (1981), Srivastava and Giles (1987), Liu (2002), Wang and Veraverbeke (2008), Ma and Ye (2010) and Wang et all (2011) discussed an efficient estimation procedure for a system of two SUR equations.

Consider a system of two SUR equations

$$
y_{i}=X_{i} \beta_{i}+\varepsilon_{i}, i=1,2
$$

where $y_{i}$ is a $T \times 1$ vector of observations on the dependent variable in the $i$ th equation, $X_{i}$ is a $T \times n_{i}$ matrix of explanatory variables in the $i$ th equation with rank $n_{i}, \beta_{i}$ is a $n_{i} \times 1$ vector of unknown parameters in the $i$ th equation, $\varepsilon_{i}$ is an $T \times 1$ vector of unobservable disturbances with

$$
E\left(\varepsilon_{i}\right)=0 \text { and } \operatorname{Cov}\left(\varepsilon_{i}, \varepsilon_{j}\right)=\sigma_{i j} I \quad(i, j=1,2)
$$

where $\Sigma=\left(\sigma_{i j}\right)$ is $2 \times 2$ an unknown positive definite matrix with $\sigma_{i j} \neq 0, I$ denotes a unit matrix. The SUR model can be expressed in the compact form as 


$$
y=X \beta+e
$$

where

$$
y=\left(\begin{array}{l}
y_{1} \\
y_{2}
\end{array}\right), X=\left(\begin{array}{cc}
X_{1} & 0 \\
0 & X_{2}
\end{array}\right), \beta=\left(\begin{array}{l}
\beta_{1} \\
\beta_{2}
\end{array}\right) \text { and } \varepsilon=\left(\begin{array}{c}
\varepsilon_{1} \\
\varepsilon_{2}
\end{array}\right) .
$$

The disturbances $\varepsilon$ has mean vector 0 and dispersion matrix $\Sigma \otimes I$, where $\otimes$ represents the kronecker product.

Generalized least squares (GLS) estimator of $\beta$

$$
\hat{\beta}_{G L S}=\left(X^{\prime}(\Sigma \otimes I)^{-1} X\right)^{-1} X^{\prime}(\Sigma \otimes I)^{-1} y .
$$

$\hat{\beta}_{G L S}$ is the best linear unbiased estimator (BLUE) of $\beta$ in the SUR model. This estimate is not a feasible estimator of $\beta$ because in general $\Sigma$ is not known. Replacing the unknown $\Sigma$ by its unrestricted estimate $S$, yields the two-stage Aitken estimator of $\beta$

$$
\hat{\beta}_{F G L S}=\left(\begin{array}{l}
\hat{\beta}_{F G L S}^{1} \\
\hat{\beta}_{F G L S}^{2}
\end{array}\right)=\left(X^{\prime}(S \otimes I)^{-1} X\right)^{-1} X^{\prime}(S \otimes I)^{-1} y .
$$

The elements $s_{i j}$ of $S$ are based on the residuals $\hat{\varepsilon}_{i}$ obtained by regressing $y_{i}$ on all the regressors in the system and are given by

$$
s_{i j}=\frac{1}{T} \hat{\varepsilon}_{i}^{\prime} \hat{\varepsilon}_{j}=\frac{1}{T} y_{i}^{\prime} \bar{P}_{Z} y_{j}(i, j=1,2)
$$

where $\bar{P}_{Z}=I-Z\left(Z^{\prime} Z\right)^{-1} Z^{\prime}, Z=\left(X_{1}, X_{2}\right)$.

Zellner (1963) assumed that the regressors in the two equations are orthogonal (as $\left.X_{1}^{\prime} X_{2}=0\right)$ and then proved that the two-stage Aitken estimator is superior to the ordinary least squares estimator (OLS). The two-stage Aitken estimate $\hat{\beta}_{F G L S}^{1}$ of $\beta_{1}$ is given as

$$
\hat{\beta}_{F G L S}^{1}=\left(X_{1}^{\prime} X_{1}\right)^{-1} X_{1}^{\prime} y_{1}-\frac{s_{12}}{s_{22}}\left(X_{1}^{\prime} X_{1}\right)^{-1} X_{1}^{\prime} y_{2}
$$

by Zellner (1963).

Revankar (1974) assumed that the regressors in the second equation are a proper subset of the regressors in the first equation i.e.,

$$
X_{1}=\left(X_{2}, L_{1}\right)
$$

where $L_{1}$ is a $\left(T \times\left(n_{1}-n_{2}\right)\right)$ matrix of $T$ observations on $\left(n_{1}-n_{2}\right)$ explanatory variables deleted from the second equation and examined in the context of a two equations system some finite sample properties of $\beta$ estimator. $\hat{\beta}_{F G L S}^{1}$ is given as 


$$
\tilde{\beta}_{F G L S}^{1}=\left(X_{1}^{\prime} X_{1}\right)^{-1} X_{1}^{\prime} y_{1}-\frac{s_{12}}{s_{22}}\left(X_{1}^{\prime} X_{1}\right)^{-1} X_{1}^{\prime}\left(I-P_{X_{2}}\right) y_{2}, \quad P_{X_{2}}=X_{2}\left(X_{2}^{\prime} X_{2}\right)^{-1} X_{2}^{\prime}
$$

by Revankar (1974). This estimator is also the special case of the two-stage covariance improved estimator proposed by Wang (1989) when there are only two linear equations in the system of seemingly unrelated regression (Ma and Ye, 2010).

In practical regression analysis, researchers often encounter the problem of multicollinearity. In case of multicollinearity we know that when the correlation matrix has one or more small eigenvalues, the estimates of the regression coefficients can be large in absolute value. The least squares estimator performs poorly in the presence of multicollinearity. One of the methods to overcome the multicollinearity problem is to consider parameter estimation in addition to the sample information such as under some exact or stochastic restrictions on the unknown parameters (Rao et al., 2008). Alkhamisi (2010) proposed two SUR type estimators based on combining the SUR ridge regression and the restricted least squares methods.

The object of the present paper is to consider the problem of multicollinearity and its statistical consequences for two seemingly unrelated regression (SUR) model when additional linear restrictions are assumed to hold. In Section 2, the restricted feasible GLS estimator is introduced under condition (1.4), and covariance matrix of an estimator is obtained in this section. In Section 3, we give a Monte Carlo experiment to compare the estimators. The conclusions of the paper are presented in Section 4.

\section{Proposing Estimator}

The availability of prior information in the form of exact linear restrictions is utilized in the estimation of the parameters of a linear regression model. If we have prior information for each equation, we predict to be useful in SUR model estimation problem. Let us assume that the prior information is such that it can be written in the form of linear equalities

$$
\begin{aligned}
& c_{1}=C_{1} \beta_{1} \\
& c_{2}=C_{2} \beta_{2}
\end{aligned}
$$

with $c_{i}$ an $s_{i}$ vector and $C_{i}$ a $s_{i} \times n_{i}$ matrix. Denote $c=\left(\begin{array}{ll}c_{1}^{\prime} & c_{2}^{\prime}\end{array}\right)^{\prime}, C=\operatorname{diag}\left(\begin{array}{ll}C_{1} & C_{2}\end{array}\right)$ and $\delta=\left(\begin{array}{l}\delta_{1} \\ \delta_{2}\end{array}\right)=\left(\begin{array}{c}c_{1}-C_{1} \beta_{1} \\ c_{2}-C_{2} \beta_{2}\end{array}\right)$. Then (2.1) can be represented as

$$
c=C \beta
$$

where $C$ is a known $s \times n$ matrix with $s=\sum_{i} s_{i}, n=\sum_{i} n_{i}$ for $i=1,2$ and $c$ is a known $s$ dimensional vector. In this article we suggest minimizing the sum of squared residuals of model (1.1) subject to (2.2). Therefore, the restricted regression is transformed into an optimization problem: 


$$
\begin{gathered}
\underset{\beta}{\arg \min }(y-X \beta)^{\prime}(\Sigma \otimes I)^{-1}(y-X \beta) \\
c=C \beta .
\end{gathered}
$$

For this optimization problem, we can apply the Lagrange multiplier method. Firstly, a Lagrangian function is introduced as follows

$$
L(\beta, \lambda)=(y-X \beta)^{\prime}(\Sigma \otimes I)^{-1}(y-X \beta)-2 \lambda^{\prime}(C \beta-c)
$$

where $\lambda$ is an $s$-dimensional vector of Lagrange multipliers. Differentiating function $L(\beta, \lambda)$ with respect to $\beta$ and $\lambda$, respectively, gives the normal equations

$$
\begin{aligned}
& \frac{1}{2} \frac{\partial L(\beta, \lambda)}{\partial \beta}=-X^{\prime}(\Sigma \otimes I)^{-1} y+X^{\prime}(\Sigma \otimes I)^{-1} X \beta-C^{\prime} \lambda=0 \\
& \frac{1}{2} \frac{\partial L(\beta, \lambda)}{\partial \lambda}=(C \beta-c)=0 .
\end{aligned}
$$

From Equations (2.3) and (2.4) we can easily obtain

$$
\hat{\beta}_{R}=\hat{\beta}_{G L S}+\left(X^{\prime}(\Sigma \otimes I)^{-1} X\right)^{-1} C^{\prime}\left(C\left(X^{\prime}(\Sigma \otimes I)^{-1} X\right)^{-1} C^{\prime}\right)^{-1}\left(c-C \hat{\beta}_{G L S}\right)
$$

where $\hat{\beta}_{R}$ is the restricted estimator. When $\Sigma$ is unknown, we use the consistent estimator of $\Sigma, S$ to obtain the feasible estimator of $\hat{\beta}_{R}$ :

$$
\hat{\beta}_{F R}=\hat{\beta}_{F G L S}+\left(X^{\prime}(S \otimes I)^{-1} X\right)^{-1} C^{\prime}\left(C\left(X^{\prime}(S \otimes I)^{-1} X\right)^{-1} C^{\prime}\right)^{-1}\left(c-C \hat{\beta}_{F G L S}\right)
$$

Thus, the restricted feasible SUR estimator of $\beta$ is given by

$$
\begin{aligned}
\hat{\beta}_{F R}= & \left(\begin{array}{c}
\left(X_{1}^{\prime} X_{1}\right)^{-1} X_{1}^{\prime} y_{1}-\frac{s_{12}}{s_{22}}\left(X_{1}^{\prime} X_{1}\right)^{-1} X_{1}^{\prime} \bar{P}_{X_{2}} y_{2} \\
\left(X_{2}^{\prime} X_{2}\right)^{-1} X_{2}^{\prime} y_{2}
\end{array}\right) \\
& +\left(\begin{array}{cc}
C_{1} K C_{1}^{\prime} P+C_{1} L C_{2}^{\prime} T & C_{1} K C_{1}^{\prime} S+C_{1} L C_{2}^{\prime} U \\
C_{2} M C_{1}^{\prime} P+C_{2} N C_{2}^{\prime} T & C_{2} M C_{1}^{\prime} S+C_{2} N C_{2}^{\prime} U
\end{array}\right) \\
& \times\left(\begin{array}{c}
c_{1}-C_{1}\left(X_{1}^{\prime} X_{1}\right)^{-1} X_{1}^{\prime} y_{1}+\frac{s_{12}}{s_{22}} C_{1}\left(X_{1}^{\prime} X_{1}\right)^{-1} X_{1}^{\prime} \bar{P}_{X_{2}} y_{2} \\
c_{2}-C_{2}\left(X_{2}^{\prime} X_{2}\right)^{-1} X_{2}^{\prime} y_{2} \\
X_{12}\left(X_{1}^{\prime} X_{1}\right)^{-1} X_{1}^{\prime} \bar{P}_{X_{2}} y_{2}+a \\
s_{22}
\end{array}\right] \\
& =\left[\begin{array}{c}
\left.\left(X_{1}^{\prime} X_{1}\right)^{-1} X_{2}^{\prime} X_{2}\right)^{-1} X_{2}^{\prime} y_{2}+b \\
\left(X_{2}+b\right.
\end{array}\right]
\end{aligned}
$$




$$
=\left(\begin{array}{l}
\hat{\beta}_{F R(1)} \\
\hat{\beta}_{F R(2)}
\end{array}\right)
$$

where

$$
\begin{aligned}
K & =\frac{1}{s_{22}}\left(X_{1}^{\prime} X_{1}\right)^{-1}\left[I+X_{1}^{\prime} P_{X_{2}} X_{1}\left(X_{1}^{\prime} X_{1}\right)^{-1}\right], \quad L=\frac{r^{2}}{s_{12}\left(1-r^{2}\right)}\left(X_{1}^{\prime} X_{1}\right)^{-1} X_{1}^{\prime} X_{2}\left(X_{2}^{\prime} X_{2}\right)^{-1} \\
M & =\frac{r^{2}}{s_{12}\left(1-r^{2}\right)}\left(X_{2}^{\prime} X_{2}\right)^{-1}\left(X_{2}^{\prime} X_{1}\right)\left(X_{1}^{\prime} X_{1}\right)^{-1}, \quad N=\frac{1}{s_{11}\left(1-r^{2}\right)}\left(X_{2}^{\prime} X_{2}\right)^{-1} \\
a & =\left[C_{1} K C_{1}^{\prime} P+C_{1} L C_{2}^{\prime} T\right]\left\{c_{1}-C_{1}\left(X_{1}^{\prime} X_{1}\right)^{-1} X_{1}^{\prime} y_{1}+\frac{s_{12}}{s_{22}} C_{1}\left(X_{1}^{\prime} X_{1}\right)^{-1} X_{1}^{\prime} \bar{P}_{X_{2}} y_{2}\right\} \text { and } \\
& +\left[C_{1} K C_{1}^{\prime} S+C_{1} L C_{2}^{\prime} U\right]\left\{c_{2}-C_{2}\left(X_{2}^{\prime} X_{2}\right)^{-1} X_{2}^{\prime} y_{2}\right\} \\
b & =\left[C_{2} M C_{1}^{\prime} P+C_{2} N C_{2}^{\prime} T\right]\left\{c_{1}-C_{1}\left(X_{1}^{\prime} X_{1}\right)^{-1} X_{1}^{\prime} y_{1}+\frac{s_{12}}{s_{22}} C_{1}\left(X_{1}^{\prime} X_{1}\right)^{-1} X_{1}^{\prime} \bar{P}_{X_{2}} y_{2}\right\} \\
& +\left[C_{2} M C_{1}^{\prime} S+C_{2} N C_{2}^{\prime} U\right]\left\{c_{2}-C_{2}\left(X_{2}^{\prime} X_{2}\right)^{-1} X_{2}^{\prime} y_{2}\right\}
\end{aligned}
$$

$\hat{\beta}_{F R(1)}$ is simplified as

$$
\begin{aligned}
\hat{\beta}_{F R(1)}= & {\left[I-\left(C_{1} K C_{1}^{\prime} P C_{1}+C_{1} L C_{2}^{\prime} T C_{1}\right)\right]\left(X_{1}^{\prime} X_{1}\right)^{-1} X_{1}^{\prime} y_{1} } \\
& -\frac{s_{12}}{s_{22}}\left[I-\left(C_{1} K C_{1}^{\prime} P C_{1}+C_{1} L C_{2}^{\prime} T C_{1}\right)\right]\left(X_{1}^{\prime} X_{1}\right)^{-1} X_{1}^{\prime} \bar{P}_{X_{2}} y_{2} \\
& -\left[C_{1} K C_{1}^{\prime} S C_{2}+C_{1} L C_{2}^{\prime} U C_{2}\right]\left(X_{2}^{\prime} X_{2}\right)^{-1} X_{2}^{\prime} y_{2} \\
& +\left[C_{1} K C_{1}^{\prime} S+C_{1} L C_{2}^{\prime} U\right] c_{2}+\left[C_{1} K C_{1}^{\prime} P+C_{1} L C_{2}^{\prime} T\right] c_{1}
\end{aligned}
$$

or

$$
\begin{aligned}
\hat{\beta}_{F R(1)}= & \left(I-D_{1}\right)\left(X_{1}^{\prime} X_{1}\right)^{-1} X_{1}^{\prime} y_{1}-\frac{s_{12}}{s_{22}}\left(I-D_{1}\right)\left(X_{1}^{\prime} X_{1}\right)^{-1} X_{1}^{\prime} \bar{P}_{X_{2}} y_{2} \\
& -D_{2}\left(X_{2}^{\prime} X_{2}\right)^{-1} X_{2}^{\prime} y_{2}+\left[C_{1} K C_{1}^{\prime} S+C_{1} L C_{2}^{\prime} U\right] c_{2}+\left[C_{1} K C_{1}^{\prime} P+C_{1} L C_{2}^{\prime} T\right] c_{1} \\
& =\left(I-D_{1}\right) \beta_{1}+\left(I-D_{1}\right)\left(X_{1}^{\prime} X_{1}\right)^{-1} X_{1}^{\prime} \varepsilon_{1}-\frac{s_{12}}{s_{22}}\left(I-D_{1}\right)\left(X_{1}^{\prime} X_{1}\right)^{-1} X_{1}^{\prime} \bar{P}_{X_{2}} \varepsilon_{2}-D_{2} \beta_{2} \\
& -D_{2}\left(X_{2}^{\prime} X_{2}\right)^{-1} X_{2}^{\prime} \varepsilon_{2}+\left[C_{1} K C_{1}^{\prime} S+C_{1} L C_{2}^{\prime} U\right] c_{2}+\left[C_{1} K C_{1}^{\prime} P+C_{1} L C_{2}^{\prime} T\right] c_{1}
\end{aligned}
$$

where $D_{1}=C_{1} K C_{1}^{\prime} P C_{1}+C_{1} L C_{2}^{\prime} T C_{1}, D_{2}=C_{1} K C_{1}^{\prime} S C_{2}+C_{1} L C_{2}^{\prime} U C_{2}$,

$$
\begin{aligned}
& P=\left(C_{1} K C_{1}^{\prime}\right)^{-1}+\left(C_{1} K C_{1}^{\prime}\right)^{-1} C_{1} L C_{2}^{\prime}\left[C_{2} N C_{2}^{\prime}-C_{2} M C_{1}^{\prime}\left(C_{1} K C_{1}^{\prime}\right)^{-1} C_{1} L C_{2}^{\prime}\right]^{-1} C_{2} M C_{1}^{\prime}\left(C_{1} K C_{1}^{\prime}\right)^{-1} \\
& S=-\left(C_{1} K C_{1}^{\prime}\right)^{-1} C_{1} L C_{2}^{\prime}\left[C_{2} N C_{2}^{\prime}-C_{2} M C_{1}^{\prime}\left(C_{1} K C_{1}^{\prime}\right)^{-1} C_{1} L C_{2}^{\prime}\right]^{-1}
\end{aligned}
$$


$T=-\left[C_{2} N C_{2}^{\prime}-C_{2} M C_{1}^{\prime}\left(C_{1} K C_{1}^{\prime}\right)^{-1} C_{1} L C_{2}^{\prime}\right]^{-1} C_{2} M C_{1}^{\prime}\left(C_{1} K C_{1}^{\prime}\right)^{-1}$

$U=\left[C_{2} N C_{2}^{\prime}-C_{2} M C_{1}^{\prime}\left(C_{1} K C_{1}^{\prime}\right)^{-1} C_{1} L C_{2}^{\prime}\right]^{-1}$. Clearly under the $\delta=0, \hat{\beta}_{F R(1)}$ estimator is $\hat{\beta}_{1}$ estimator.

We investigate the efficiency of the restricted feasible SUR estimator of $\beta_{1}$, as compared to the unrestricted estimator in Revankar (1974). The expected value of (2.8) is equal to $E\left(\hat{\beta}_{F R(1)}\right)=\left(I-D_{1}\right) \beta_{1}-D_{2} \beta_{2}+\left[C_{1} K C_{1}^{\prime} S+C_{1} L C_{2}^{\prime} U\right] c_{2}+\left[C_{1} K C_{1}^{\prime} P+C_{1} L C_{2}^{\prime} T\right] c_{1}$.

This means that $\hat{\beta}_{F R(1)}$ is biased estimator of $\beta_{1}$. Thus, the covariance matrix for (2.8) is given by

$$
\operatorname{Cov}\left(\hat{\beta}_{F R(1)}\right)=E\left(\hat{\beta}_{F R(1)}-E\left(\hat{\beta}_{F R(1)}\right)\right)\left(\hat{\beta}_{F R(1)}-E\left(\hat{\beta}_{F R(1)}\right)\right)^{\prime}=\sum_{i=1}^{9} Q_{i}
$$

where

$$
\begin{aligned}
& Q_{1}=E\left(\left(I-D_{1}\right) \varepsilon_{1} \varepsilon_{1}^{\prime}\left(I-D_{1}\right)^{\prime}\right) \\
& Q_{2}=-E\left(\frac{s_{12}}{s_{22}}\left(I-D_{1}\right) \varepsilon_{1} \varepsilon_{2}^{\prime} \bar{P}_{X_{2}} X_{1}\left(X_{1}^{\prime} X_{1}\right)^{-1}\left(I-D_{1}\right)^{\prime}\right) \\
& Q_{3}=-E\left(\left(I-D_{1}\right) \varepsilon_{1} \varepsilon_{2}^{\prime} X_{2}\left(X_{2}^{\prime} X_{2}\right)^{-1} D_{2}^{\prime}\right) \\
& Q_{4}=-E\left(\frac{s_{12}}{s_{22}}\left(I-D_{1}\right)\left(X_{1}^{\prime} X_{1}\right)^{-1} X_{1}^{\prime} \bar{P}_{X_{2}} \varepsilon_{2} \varepsilon_{1}^{\prime}\left(I-D_{1}\right)^{\prime}\right) \\
& Q_{5}=E\left(\frac{s_{12}^{2}}{s_{22}^{2}}\left(I-D_{1}\right)\left(X_{1}^{\prime} X_{1}\right)^{-1} X_{1}^{\prime} \bar{P}_{X_{2}} \varepsilon_{2} \varepsilon_{2}^{\prime} \bar{P}_{X_{2}} X_{1}\left(X_{1}^{\prime} X_{1}\right)^{-1}\left(I-D_{1}\right)^{\prime}\right), \\
& Q_{6}=E\left(\frac{s_{12}}{s_{22}}\left(I-D_{1}\right)\left(X_{1}^{\prime} X_{1}\right)^{-1} X_{1}^{\prime} \bar{P}_{X_{2}} \varepsilon_{2} \varepsilon_{2}^{\prime} X_{2}\left(X_{2}^{\prime} X_{2}\right)^{-1} D_{2}^{\prime}\right) \\
& Q_{7}=-E\left(D_{2}\left(X_{2}^{\prime} X_{2}\right)^{-1} X_{2}^{\prime} \varepsilon_{2} \varepsilon_{1}^{\prime}\left(I-D_{1}\right)^{\prime}\right) \\
& Q_{8}=E\left(\frac{s_{12}}{s_{22}} D_{2}\left(X_{2}^{\prime} X_{2}\right)^{-1} X_{2}^{\prime} \varepsilon_{2} \varepsilon_{2}^{\prime} \bar{P}_{X_{2}} X_{1}\left(X_{1}^{\prime} X_{1}\right)^{-1}\left(I-D_{1}\right)^{\prime}\right)
\end{aligned}
$$

and

$$
Q_{9}=E\left(D_{2}\left(X_{2}^{\prime} X_{2}\right)^{-1} X_{2}^{\prime} \varepsilon_{2} \varepsilon_{2}^{\prime} X_{2}\left(X_{2}^{\prime} X_{2}\right)^{-1} D_{2}^{\prime}\right)
$$

Following Zellner (1963), Revankar (1974) and Liu (2002), $\frac{s_{12}}{s_{22}}$ obeys a Pearson Type VII or Student " $t$ " distribution with

$$
E\left(\frac{s_{12}}{s_{22}}\right)=\frac{\sigma_{12}}{\sigma_{22}}
$$


and

$$
E\left[\left(\frac{s_{12}}{s_{22}}\right)^{2}\right]=\frac{\sigma_{11}}{\sigma_{22}} \rho^{2}+\frac{\sigma_{11}}{\sigma_{22}} \frac{1-\rho^{2}}{T-n_{1}-2}
$$

where $n_{1}=\operatorname{rank}\left(X_{1}\right)$. Using these results we can find that

$$
\begin{aligned}
& Q_{1}=\sigma_{11}\left(I-D_{1}\right)\left(I-D_{1}\right)^{\prime} \\
& Q_{2}=-\frac{\sigma_{12}^{2}}{\sigma_{22}}\left(I-D_{1}\right) \bar{P}_{X_{2}} X_{1}\left(X_{1}^{\prime} X_{1}\right)^{-1}\left(I-D_{1}\right)^{\prime} \\
& Q_{3}=-\sigma_{12}\left(I-D_{1}\right) X_{2}\left(X_{2}^{\prime} X_{2}\right)^{-1} D_{2}^{\prime} \\
& Q_{4}=-\frac{\sigma_{12}^{2}}{\sigma_{22}}\left(I-D_{1}\right)\left(X_{1}^{\prime} X_{1}\right)^{-1} X_{1}^{\prime} \bar{P}_{X_{2}}\left(I-D_{1}\right)^{\prime} \\
& Q_{5}=\sigma_{11}\left(\rho^{2}+\frac{1-\rho^{2}}{T-n_{1}-2}\right)\left(I-D_{1}\right)\left(X_{1}^{\prime} X_{1}\right)^{-1} X_{1}^{\prime} \bar{P}_{X_{2}} X_{1}\left(X_{1}^{\prime} X_{1}\right)^{-1}\left(I-D_{1}\right)^{\prime} \\
& Q_{6}=\sigma_{12}\left(I-D_{1}\right)\left(X_{1}^{\prime} X_{1}\right)^{-1} X_{1}^{\prime} \bar{P}_{X_{2}} X_{2}\left(X_{2}^{\prime} X_{2}\right)^{-1} D_{2}^{\prime} \\
& Q_{7}=-\sigma_{21} D_{2}\left(X_{2}^{\prime} X_{2}\right)^{-1} X_{2}^{\prime}\left(I-D_{1}\right)^{\prime} \\
& Q_{8}=\sigma_{12} D_{2}\left(X_{2}^{\prime} X_{2}\right)^{-1} X_{2}^{\prime} \bar{P}_{X_{2}} X_{1}\left(X_{1}^{\prime} X_{1}\right)^{-1}\left(I-D_{1}\right)^{\prime}
\end{aligned}
$$

and

$$
Q_{9}=\sigma_{22} D_{2}\left(X_{2}^{\prime} X_{2}\right)^{-1} D_{2}^{\prime}
$$

From (2.10) and (a)-(i), we get

$$
\begin{aligned}
& \operatorname{Cov}\left(\hat{\beta}_{F R(1)}\right)=\left(I-D_{1}\right) \\
& \times\left[\sigma_{11} I-\frac{\sigma_{12}^{2}}{\sigma_{22}} \bar{P}_{X_{2}} X_{1}\left(X_{1}^{\prime} X_{1}\right)^{-1}-\frac{\sigma_{12}^{2}}{\sigma_{22}}\left(X_{1}^{\prime} X_{1}\right)^{-1} X_{1}^{\prime} \bar{P}_{X_{2}}+\sigma_{11}\left(\rho^{2}+\frac{1-\rho^{2}}{T-n_{1}-2}\right)\left(X_{1}^{\prime} X_{1}\right)^{-1} X_{1}^{\prime} \bar{P}_{X_{2}} X_{1}\left(X_{1}^{\prime} X_{1}\right)^{-1}\right] \\
& \times\left(I-D_{1}\right)^{\prime}-\left(I-D_{1}\right)\left[\sigma_{12} X_{2}\left(X_{2}^{\prime} X_{2}\right)^{-1}\right] D_{2}^{\prime}-D_{2}\left[\sigma_{21}\left(X_{2}^{\prime} X_{2}\right)^{-1} X_{2}^{\prime}\right]\left(I-D_{1}\right)^{\prime}+\sigma_{22} D_{2}\left(X_{2}^{\prime} X_{2}\right)^{-1} D_{2}^{\prime} \\
& =\sigma_{11}\left(I-D_{1}\right)\left[I-2 \rho^{2} \bar{P}_{X_{2}} X_{1}\left(X_{1}^{\prime} X_{1}\right)^{-1}+\left(\rho^{2}+\frac{1-\rho^{2}}{T-n_{1}-2}\right)\left(X_{1}^{\prime} X_{1}\right)^{-1} X_{1}^{\prime} \bar{P}_{X_{2}} X_{1}\left(X_{1}^{\prime} X_{1}\right)^{-1}\right]\left(I-D_{1}\right)^{\prime} \\
& -2 \sigma_{12}\left(I-D_{1}\right) X_{2}\left(X_{2}^{\prime} X_{2}\right)^{-1} D_{2}^{\prime}+\sigma_{22} D_{2}\left(X_{2}^{\prime} X_{2}\right)^{-1} D_{2}^{\prime}
\end{aligned}
$$

or

$$
\begin{aligned}
& =\sigma_{11}\left(I-D_{1}\right)\left(I-\rho^{2} A\right)^{\prime}\left(I-\rho^{2} A\right)\left(I-D_{1}\right)^{\prime} \\
& +\sigma_{11}\left(1-\rho^{2}\right)\left(\rho^{2}+\frac{1}{T-n_{1}-2}\right)\left(I-D_{1}\right) A^{\prime} A\left(I-D_{1}\right)^{\prime} \\
& -2 \sigma_{12}\left(I-D_{1}\right) X_{2}\left(X_{2}^{\prime} X_{2}\right)^{-1} D_{2}^{\prime}+\sigma_{22} D_{2}\left(X_{2}^{\prime} X_{2}\right)^{-1} D_{2}^{\prime}
\end{aligned}
$$

where $A=\bar{P}_{X_{2}} X_{1}\left(X_{1}^{\prime} X_{1}\right)^{-1}$ 
We now compare the $\hat{\beta}_{F R(1)}$ estimator with $\hat{\beta}_{F G L S}^{1}$ estimator and $\tilde{\beta}_{F G L S}^{1}$ estimator by the matrix mean square (MSE) criterion. The nonnegativeness of mse among these estimators cannot be easily seen. In order to observe the performance of these estimators, we will prepare a simulation study next section.

\section{The Simulation Study}

In this section the performance of the proposed restricted feasible SUR estimator of $\beta$ is examined via Monte Carlo simulations. This simulation study is partly based on Alkhamisi (2010). In all simulations, the explanatory variable $X_{1}$ is generated from $\operatorname{MVN}_{4}\left(0, \Sigma_{X}\right)$ with $\operatorname{diag}\left(\Sigma_{X}\right)=1$ and off $-\operatorname{diag}\left(\Sigma_{X}\right)=\rho_{X}$. Three different sets of correlations namely $\rho_{X}=0.8,0.9$ and 0.99 are considered. We consider that $X_{2}$ is subset of $X_{1}$. The random errors are generated from $\operatorname{MVN}_{3}\left(0, \Sigma_{e}\right)$ with parameters $\operatorname{diag}\left(\Sigma_{e}\right)=1$ and $\operatorname{off}-\operatorname{diag}\left(\Sigma_{e}\right)=\rho_{e}$ and correlation coefficients $\rho_{e}=0.35$ and 0.8 . $\beta$ parameter vectors are chosen arbitrarily such as suitable dimensional $\mathbf{1}$ vector. Observations on the dependent variable are determined with the following equation,

$$
y_{t i}=\sum_{j=1}^{n_{i}} x_{t i j} \beta_{i j}+e_{t i}, t=1, \ldots, T ; i=1,2
$$

where $x_{t i l=1}$. To study the effect of small and large sample on the properties of estimators of $\beta$ we considered samples of sizes 15 and 100 for small and large samples respectively. The restriction matrices are given in Table 1 .

Table 1: Linear restrictions for each equations

\begin{tabular}{ccccccc}
\hline$C_{1}$ & & \multicolumn{5}{c}{$C_{2}$} \\
\hline 7 & 0 & 3 & 0 & 0 & 4 & 0 \\
0 & 4 & 0 & 0 & 2 & 0 & 0 \\
3 & 0 & 0 & 1 & 1 & 0 & 3 \\
\hline
\end{tabular}

For each choice of $\rho_{X}, \rho_{e}$ and $T$ the experiment is replicated 1000 times and then the MSEs for the estimators are calculated as follows

$$
\operatorname{MSE}\left(\alpha^{*}\right)=\frac{1}{1000} \sum_{i=1}^{1000}\left(\alpha_{i}^{*}-\alpha\right)^{\prime}\left(\alpha_{i}^{*}-\alpha\right)
$$

where $\alpha_{i}^{*}$ denotes the estimated parameter in the $i$-th simulation.

MATLAB R2009 is used for the simulation. From the simulation results shown in Table 2, we can see that FGLS estimator performs the worst among all estimators in terms of scalar MSE. The performance of RF estimator outperforms other estimators in terms of scalar MSE except for high correlation among the explanatory variables and among the 
equations. The increase in the correlation among the explanatory variables leads to an increase in the scalar MSEs for all the estimators. We observed that as the correlation among equations increases, the scalar MSEs of the estimators increases.

Table 2: Estimated scalar MSE of the FGLS, Revankar and RF estimators

\begin{tabular}{cccccc}
\hline$T$ & $\rho_{X}$ & $\rho_{e}$ & FGLS & Revankar & RF \\
\hline 15 & 0.8 & 0.35 & 0,060677 & 0,015991 & 0,009497 \\
& & 0.8 & 0,216745 & 0,016083 & 0,012344 \\
& 0.9 & 0.35 & 0,229289 & 0,015995 & 0,009849 \\
& 0.99 & 0.35 & 7,949551 & 0,016026 & 0,014788 \\
& & 0.8 & 2,962609 & 0,016424 & 0,011265 \\
& 0.8 & 0.35 & 0,028764 & 0,016004 & 0,009313 \\
& & 0.8 & 0,180810 & 0,016264 & 0,015973 \\
& 0.9 & 0.35 & 0,064938 & 0,016006 & 0,009215 \\
& & 0.8 & 4,398776 & 0,016321 & 0,014290 \\
& 0.99 & 0.35 & 1,204430 & 0,016113 & 0,009671 \\
& & 0.8 & 5,389418 & 0,018659 & 0,024309 \\
\hline
\end{tabular}

\section{Conclusion remarks}

In this article, we proposed a restricted feasible SUR estimator for the vector of parameters in two seemingly unrelated regression models when additional linear restrictions on the parameter vector are assumed to hold. The restricted feasible estimator of the $\beta$ parameter vector is then compared with the FGLS estimator and the estimator proposed by Revankar (1974) in terms of MSE criterion. The investigation has been done by means of Monte Carlo simulations. The results have shown that our proposed estimator produce smaller MSEs.

\section{References}

1. Alkhamisi, M.A. (2010). Simulation study of new estimators combining the SUR ridge regression and the restricted least squares methodologies, Statistical Papers, 51(3), 651-672.

2. Kariya, T. (1981). Tests for the independence between two seemingly unrelated regression equations, The Annals of Statistics, 9(2), 381-390.

3. Liu, A.Y. (2002). Efficient estimation of two seemingly unrelated regression equations, Journal of Multivariate Analysis, 82, 445-456.

4. Ma, T.F. and Ye, R. (2010). Efficient improved estimation of the parameters in two seemingly unrelated regression models, Journal of Statistical Planning and Inference, 140, 2749-2754. 
5. Rao, C. R., Toutenburg, H., Shalabh and Heumann, C. (2008). Linear Models and Generalizations: Least Squares and Alternatives, Springer, Berlin.

6. Revankar, N.S. (1974). Some finite sample results in the context of two seemingly unrelated regression equations, Journal of the American Statistical Association, 69, 187-190.

7. Srivastava, V. and Giles, D. (1987). Seemingly Unrelated Regression Equations Models, Marcel Dekker, New York.

8. Wang, S.G. (1989). A new estimate of regression coefficients in seemingly unrelated regression system, Science in China Series A, 32, 808-816.

9. Wang, L. and Veraverbeke, N. (2008). MSE superiority of Bayes and empirical Bayes estimators in two generalized seemingly unrelated regressions, Statistics and Probability Letters, 78, 109-117.

10. Wang, L., Lian, H. and Singh, R.S. (2011). On efficient estimators of two seemingly unrelated regressions, Statistics and Probability Letters, 81, 563-570.

11. Zellner, A. (1963). Estimators of seemingly unrelated regressions: Some exact finite sample results, Journal of the American Statistical Association, 58, 977-992.

12. Zellner, A. (1962). An efficient method of estimating seemingly unrelated regressions and tests for aggregation bias, Journal of the American Statistical Association, 57, 348-368. 